\title{
PRUEBA COLECTIVA SIN PRODUCCIÓN ORAL PARA LA EVALUACIÓN DE LOS PROCESOS DE RECONOCIMIENTO DE LA PALABRA ESCRITA. PIPE
}

\author{
Virginia González Santamaría \\ Universidad de Salamanca \\ virginia_gonzalez_santamaria@usal.es
}

Fecha de Recepción: 21 Enero 2019

Fecha de Admisión: 30 Abril 2019

\section{RESUMEN}

El trabajo tiene por objetivo la presentación de una prueba de evaluación de los procesos de reconocimiento de la palabra escrita en estudiantes de tercero de infantil y primer y segundo curso de educación primaria. Esta prueba, de aplicación colectiva, está diseñada para evaluar a todo tipo de alumnado, con y sin discapacidad, con y sin problemas del lenguaje, pues se resuelve sin necesidad de dar una respuesta oral por parte del estudiante. El modelo teórico en el que se basa la prueba establece que la comprensión lectora se define por la combinación de dos habilidades, las no específicas de la lectura, comunes a la lengua oral (sintaxis y vocabulario, principalmente), y las habilidades específicas de la misma, aquellas que solo se ponen en marcha exclusivamente cuando leemos y escribimos palabras, siendo una de las más importantes los Procesos de Reconocimiento de la Palabra Escrita. Para que un lector sea considerado experto, es necesario que el funcionamiento de estos procesos se realice con fluidez, es decir, con precisión, (sin cometer errores), y con automaticidad, esto es, sin consumir demasiados recursos cognitivos en estos procesos y poder usarlos en procesos superiores como la comprenisón. Para evaluar la fluidez en la lectura de palabras en castellano disponemos de varias pruebas de aplicación individual y que requieren una respuesta oral por parte del estudiante. Por este motivo, la prueba que se presenta se compone de 60 ítems representados por un dibujo y tres opciones de respuesta, la palabra correcta y dos distractrores ortográficos que han de completarse (el mayor número de ellos) en tres minutos. A medida que se avanza en la prueba aumenta la complejidad semántica de los ítems. Finalmente, se discute la importancia de utilizar pruebas como esta para la prevención y diagnóstico tempranos de las dificultades a este nivel.

Palabras clave: lectura; dificultades de aprendizaje; modelos proactivos; respuesta educativa; educación infantil y primaria 


\title{
PRUEBA COLECTIVA SIN PRODUCCIÓN ORAL PARA LA EVALUACIÓN DE LOS PROCESOS DE RECONOCIMIENTO DE LA PALABRA ESCRITA. PIPE
}

\begin{abstract}
Collective proof without oral production for the evaluation of the processes of recognition of the written word. PIPE. The aim of this work is to present a task of evaluation of the processes of recognition of the written word in third-year of Pre-school education and first and second years of Primary education. This task, of collective application, is designed to evaluate different students, with and without disabilities, with and without language difficulties, since it is resolved without the need for an oral response from the student. The theoretical model in which the task is based establishes that reading comprehension is defined by the combination of two skills, non-specific reading skills, common to oral language (syntax and vocabulary, mainly), and specific skills of it, those that only start up when we read and write words, being one of the most important Processes of Recognition of the Written Word. For a reader to be considered an expert, it is necessary that the operation of these processes be carried out fluently, that is, with precision, (without making mistakes), and with automaticity, that is, without consuming too many cognitive resources in these processes and be able to use them in higher processes such as reading comprenhension. To evaluate the fluency in the reading of words in Spanish we have several tasks of individual application and that require an oral response by the student. For this reason, the task that is presented consists of 60 items represented by a drawing and three response options, the correct word and two orthographic distracters to be completed (the largest number of them) in three minutes. As the test progresses, the semantic complexity of the items increases. Finally, the importance of using tasks like this for the prevention and early diagnostic of difficulties at this level is discussed.

Keywords: reading; learning disabilities; proactive models; educational response; pre-school; primary school
\end{abstract}

\section{ANTECEDENTES}

“No sé cómo aprendí a leer, sólo recuerdo mis primeras lecturas" (Rousseau).

Con estas palabras ya Rousseau en el siglo XVIII puso de manifiesto que el aprendizaje de la lectura, al igual que todas las habilidades cognitivas, cuando se dominan, son procesos simples que aparentemente no requieren esfuerzo. Hoy en día disponemos de evidencias científicas que indican que la lectura no es un "acto natural', sino que nuestro cerebro tiene que aprender a leer y necesita años de trabajo para desarrollar unos mecanismos que se especialicen en decodificar los códigos escritos y transformarlos en lenguaje (Defior, 2014). Las personas adquirimos la lengua materna mediante un proceso natural, por el hecho de estar inmersos en un contexto adecuado desde el punto de vista lingüístico, sin embargo, aprender a leer consiste en desarrollar, por medio de una enseñanza temprana, explícita y sistemáctica, un mecanismo que ayude a identificar todas las palabras que el lector conoce oralmente (Alegría, 2006; Alegría Carrillo y Sánchez, 2005). Aprender a leer, y más importante aún, adquirir buenos niveles lectores en sociedades como la nuestra, donde la mayor parte de la información y el acceso al conocimiento se realiza a través de la lengua escrita, supone un problema social para aquellas personas que, tras un período de enseñanza, por diversas razones, no aprenden a leer (Morais, 1994).

En este contexto, para decodificar el código escrito es necesario poner en marcha las reglas de correspondencia grafema-fonema, es decir, asignar un fonema al estímulo visual (Defior, 2014). Este es un requisito indispensable para leer, pero no es el único. Para comprender el proceso lector, es necesario disponer de un marco teórico que describa cuáles son las habilidades implicadas, cómo funcionan y qué problemas pueden surgir para que el aprendizaje de la lectura no se produzca. El marco teórico en el que se desarrolla este trabajo se basa en la concepción psicolingüística de 
la lectura, donde se intenta dar respuesta a cuestiones relacionadas con ¿cómo se aprende a leer? ¿Qué habilidades tiene que tener un lector para considerarse experto? En ella se propone que aprender a leer es una actividad compleja que requiere poner en marcha habilidades de tipo congnitivo, lingüístico y metacognitivo (Alegría, 1985; Morais, 1994). Por tanto, desde esta perspectiva, la comprensión lectora se define por la combinación de dos habilidades: por un lado, los procesos no específicos de la lectura, comunes a la lengua oral, y por otro, los procesos específicos de la lectura, aquellas habilidades que únicamente se ponen en marcha cuando se lee y se escribe palabras (Alegría, 1985, 2006, Morais, 1994). Entre las primeras, se encuentran principalmente la morfosintaxis, es decir, el conocimiento de las reglas morfológicas y sintácticas de una lengua, y el vocabulario, entendido este como el conjunto de palabras que una persona conoce y emplea, y en las segundas, los Procesos de Reconocimiento de la Palabra Escrita (Figura 1).

Figura 1. Componentes de la comprensión del leguaje escrito (Tomado de Morais, 1994, p.185)

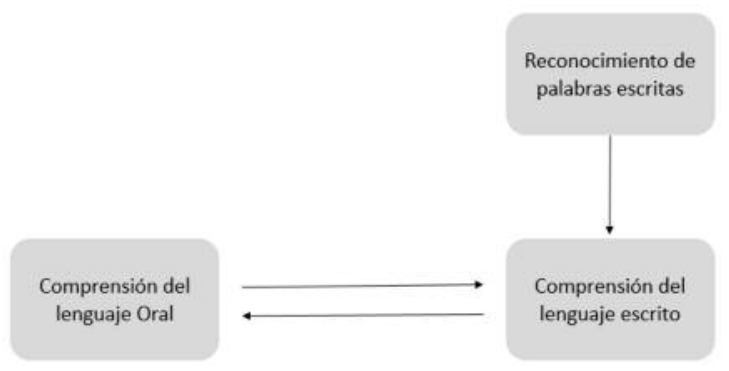

Este marco teórico fue ya planteado por Gough y Tunmer (1986) y Hoover y Gough (1990) denominádolo Modelo Simple de Lectura. Estos autores utilizaron el término "simple" porque conciben el proceso como la combinación únicamente de dos elementos (Comprensión Oral y Procesos de Reconocimiento de la Palabra Escrita), no porque el proceso lector en sí sea simple. Por ello, y para poder identificar los aspectos subyacentes a estos dos elementos, Ripoll y Aguado (2015) indican que esta forma de concebir la lectura pretende ser un modelo didáctico en el que se ponga de manifiesto qué se necesita para mejorar la comprensión lectora y cómo intervenir de manera proactiva para que esas dificultades de aprendizaje de la lectura no avancen de forma significativa y repercutan en aprendizajes posteriores (Modelos de Respuesta a la Intervención, Jiménez et al., 2011). Estos modelos tienen como eje central un poceso multinivel, esto es, se establecen tres niveles caracterizados por el tipo de enseñanza, el número de estudiantes a los que va dirigido y la respuesta que estos tienen a la intervención. El primer nivel, centrado en la enseñanza general de la lectura, evalúa e interviene con todos los estudiantes, y, aquellos con dificultades en las habilidades específicas de la lectura, llevarán un seguimiento más concreto basado en evaluaciones más continuadas. El segundo nivel, de intervención, está formado por aquellos estudiantes que en el primer nivel no han progresado lo suficiente tras la intervención, de manera que se plantea una intervención en pequeño grupo con una duración aproximada de media hora basada en los elementos clave de aprendizaje lector: conciencia fonémica, procesamiento fonológico, fluidez, vocabulario y comprenisón (NICHD, 2000). Por último, el tercer nivel, de instrucción intensiva, lo conforman aquellos estudiantes que tras la intervención del segundo nivel no muestran la mejora que cabría esperar. En este nivel la intervención, aunque sigue siendo en grupo, es más individualizada y tiene una dura- 


\section{PRUEBA COLECTIVA SIN PRODUCCIÓN ORAL PARA LA EVALUACIÓN DE LOS PROCESOS DE RECONOCIMIENTO DE LA PALABRA ESCRITA. PIPE}

ción aproximada de una hora. Finalizado el proceso, aquellos estudiantes que han recibido una intervención adecuada a sus necesidades y no han tenido un nivel de progreso acorde a lo esperado, son considerados estudiantes con dificultades específicas de aprendizaje y deben derivarse a los equipos educativos específicos. A continuación se desarrollarán de una manera más concreta los procesos específicos de la lectura, por ser estos el objeto de evaluación de la prueba que se presenta en este trabajo.

Los procesos de reconocimiento de la palabra escrita son habilidades en las que la fonología de la lengua oral tiene un papel importante puesto que perminte conectar las letras que componen la palabra con su significado previamente establecido, siendo esta, una actividad que no sirve más que para leer (Alegría, 2003; Morais, 1994). Así, la identificación de la palabra escrita implica, por un lado, asignar sonidos a los signos gráficos, es decir, aplicar las reglas de correspondencia grafemafonema, para su posterior identificación ortográfica, y, a partir de ahí, acceder a los conocimientos que se tenga sobre la palabra (léxicos, semánticos, sintácticos y pragmáticos) que está almacenada en una estructura mental Ilamada léxico interno (Alegría, 1985, 2003; Cuetos y Valle, 1988; Defior, 2014; Morais, 1994; Perfetti y Sandak, 2000).

Son muchos los modelos que explican cómo se accede al léxico interno. En la actualidad, uno de los más aceptados es el modelo de la Doble Ruta, que establece que para acceder al léxico interno disponemos de dos vías: la vía léxica o directa y la vía fonológica o indirecta (Coltheart, 1985; Coltheart, Curtis y Haller, 1993; Coltheart, Rastle, Perry, Lang- don y Ziegler, 2001). La vía directa 0 ruta léxica es aquella que permite leer palabras familiares y su funcionamiento consiste en emparejar la imagen visual de la palabra escrita con la representación ortográfica almacenada en léxico interno, sin que intervenga la fonología (Coltheart, 2005). Para poder leer y escribir palabras por esta vía, es necesario haber visto y escrito muchas veces una palabra de forma correcta. Esta ruta, además, permite escribir palabras con ortografía arbitraria, es decir, que un fonema, por ejemplo, /b/ puede tener varios grafemas que lo represente: la letra b y la letra v. A pesar de poder disponer de una elevada cantidad de entradas ortográficas que nos permitan leer las palabras de forma automática, esto es, sin consumir demasiados recursos cognitivos de atención y memoria, (Coltheart, 2006, establece que se pueden tener incluso 20.000 palabras en el léxico interno) continuamente el lector se enfrenta a nuevas palabras o palabras que no tienen ningún significado (pseudoplabras). Para este tipo de palabras es necesario otro mecanismo: La vía indirecta o ruta fonológica. Esta ruta utiliza la mediación de la lengua oral, concretamente de la fonología, para acceder al significado de la palabra. En este procedimiento no se empareja la palabra escrita con el significado existente en el léxico interno, sino que lo que se empareja es la versión fonológica de la palabra, es decir, cuando se lee 0 escribe la palabra, esta se segmenta en unidades mínimas (sílabas y fonemas) y a partir de ellas se crea, mediante reglas de correspondencia grafema-fonema o fonema-grafema (en función de la tarea que se realice, lectura o escritura, respectivamente) la versión fonológica de la misma, la cual sí permite acceder al significado y a todos los conocimientos que se tengan almacenados. Estas dos vías han de ponerse en marcha de forma simultánea para conseguir una lectura fluida desde las primeras etapas educativas. Castejón, González-Pumariega y Cuetos (2015) definen esta fluidez lectora como un proceso que ha de ser preciso (sin cometer errores) y automático (sin consumir demasiados recursos cognitivos). El estudio longitudinal realizado por Castejón et al., (2011) sobre la adquisición de la fluidez lectora puso de manifiesto que se adquiere antes la precisión que la automaticidad. Los problemas de automatización provocan un reconocimiento torpe y lento de las palabras que puede llegar afectar a la comprensión lectora (Wolf y Bowers, 2000).

Dada la importancia que tiene presentar unas buenas habilidades específicas de la lectura para facilitar un adecuado proceso lector, resulta imprescindible disponer de pruebas que permitan eva- 
luar estos procesos lo antes posible y en todos los alumnos. En nuestro idioma, el castellano, disponemos de diferentes pruebas que evalúan estos aspectos, alguna de ellas son las siguientes: TALE, Test de análisis de la lectoescritura (Toro y Cervera, 1980) que evalúa la precisión y velocidad en estudiantes de 6 a 10 años, EMLE (Toro, Cervera y Ruiz, 2002), la versión revisada de TALE, PROLEC-R (Cuetos, Rodríguez y Ruano, 2007), que evalúan los procesos de comprensión del material escrito en estudiantes de 6 a 12 años. Estas pruebas son de aplicación individual, requieren de producción oral por parte del alumno y están baremadas a partir de los 6 años, por ello, se presenta la prueba PIPE, que como se describirá más adelante, es una prueba de aplicación colectiva, no requiere de respuesta oral por parte del alumnado (permitiendo evaluar también a estudiantes con discapacidad y/o problemas del lenguaje) y puede aplicarse en el último curso de educación infantil (5 años).

\section{OBJETIVOS}

El objetivo de este trabajo ha sido presentar una prueba para la evaluación de los procesos de reconocimiento de la palabra escrita en estudiantes de tercer curso de Educación Infantil y primero y segundo de Educación Primaria.

\section{PARTICIPANTES}

La muestra con la que se presenta este estudio está formada por 104 estudiantes de tercer curso de educación infantil y 452 estudiantes de educación primaria de diferentes provincias de Castilla y León. Todos ellos estaban matriculados en el curso correspondiente a su edad cronológica y ninguno de ellos tenía ningún tipo de discapacidad ni dificultades de aprendizaje diagnosticadas. El grupo de estudiantes de educación infantil tiene una edad media de 5.86 años (rango: de 5.42 a 6.33 y DT $=.28$ ). El grupo de estudiantes de educación primaria lo conforman 223 estudiantes de primer curso (Edad media = 7 años, rango: de 6.5 a 8.25 años, DT = .33) y 229 estudiantes de segundo curso (Edad media $=7.99$ años, rango: de 7.5 a 9.08 años, DT $=.33$ ).

\section{INSTRUMENTO Y PROCEDIMIENTO}

El instrumento de Evaluación utilizado para la evaluación de los Procesos de Reconocimiento de la Palabra Escrita ha sido la prueba PIPE. Esta prueba se puede obtener de forma gratuita en la siguiente dirección: https://complydis.usal.es/. Es una prueba de aplicación colectiva que está diseñada para evaluar a todo tipo de alumnado, con y sin discapacidad, con y sin problemas del lenguaje oral y que se resuelve sin necesidad de dar una respuesta oral por parte del estudiante, permitiendo, a su vez, diferenciar sin dificultad alguna un error de lectura de un error de producción.

La prueba se compone de 60 ítems en formato dibujo que representan el nombre de objetos comunes y tres palabras escritas a la derecha de cada dibujo (Figura 2). El estudiante debe elegir, marcando con un círculo, entre las tres palabras propuestas, aquella que representa el nombre del dibujo. Se dispone de tres minutos para completar el mayor número de ítems.

Figura 2. Ejemplos prueba PIPE

Haz un circulo en la palabra que represente el dibujo:
balcón
bastón


Las opciones de respuesta presentadas se han elaborado siguiendo las siguientes premisas:

Mantenimiento de la estuctura visual de la palabra. Todas las opciones de respuesta (la palabra correcta y los dos distractores) mantienen la misma estructura visual: suelen comenzar y terminar por las mismas grafias y se intenta que tengan la misma longitud (véase Figura 2).

La palabra correcta y los distractores son de la misma frecuencia, establecidos a través del diccionario de frecuencias de Martínez y García (2004).

Todos los ítems tienen entre una y cuatro sílabas y tanto la palabra correcta como los distractores mantienen la misma estructura silábica.

Los estudiantes fueron evaluados, de forma conjunta y, previo al comienzo de la prueba, esta se explicó tantas veces como fue necesario usando los ejemplos de la misma (ver Figura 2) para asegurar la comprensión de la tarea. El procedimiento aplicado para la corrección de la prueba es el número de aciertos menos el número de errores dividido entre dos para controlar el factor azar (A(E/2). La fiabilidad de la prueba evaluada con el alpha de Cronbach y la prueba de mitades indican valores elevados (alpha $=.994 ; r=.984)$.

La prueba PIPE, está siendo usada por la Consejería de Educación de la Junta de Castilla y León junto a cuatro pruebas de la batería PEALE (Pruebas de Evaluación Analítica de la Lengua Escrita) (Domínguez, Alegría, Carrillo y Soriano, 2013) como screening para la detección de dificultades de aprendizaje vinculadas a la lectura dentro de la línea estratégica 2 del II Plan de Atención a la Diversidad 2015-2020, cuyo objetivo es "mejorar los procesos de prevención, detección e intervención temprana de las necesidades educativas del alumnado". El screening está formado, por tanto, por una prueba de lectura global (TECLE, Carrillo y Marín, 1997), una prueba de Decisión Ortográfica y dos subpruebas de conciencia metafonológica: la subprueba de sílabas la subprueba de fonemas.

\section{RESULTADOS}

El objetivo de este trabajo ha sido presentar una prueba para la evaluación de los procesos de reconociminento de la palabra escrita en estudiantes del último curso de Educación Infantil y de los dos primeros cursos de Educación Primaria.

Figura 3. Resultados PIPE tercer curso de Educación Infantil Nota: la línea horizontal representa el valor promedio obtenido por el grupo

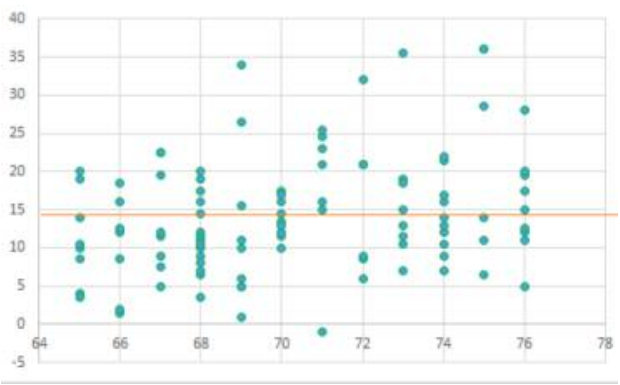

Los resultados muestran que la prueba es válida para la evaluación de los procesos de reconocimiento de la palabra escrita y, también, para la identificación de dificultades iniciales de la lectura en aquellos estudiantes con los se inicia el proceso de enseñanza del código alfabético y de la lectura. 
Como se observa en la Figura 3, los estudiantes de educación infantil obtienen resultados que oscilan entre -1 y 36 puntos. La media obtenida para este grupo es de 14.26 puntos (DT $=7.68$ ), indicando por tanto, que el $57.7 \%$ de los participantes de tercer curso de educación infantil obtenienen puntuaciones inferiores a la misma, estudiantes, por tanto, objeto de especial atención para una temprana intervención educativa.

Teniendo en cuenta los resultados obtenidos en los cursos de educación primaria, se observa una clara evolución en los mismos, llegando a obtener una puntuación media de 33.26 puntos (DT $=13.7)$ en el primer curso de educuación primaria y de 46.73 puntos $(D T=11.91)$ en el segundo curso.

Figura 4. Resultados PIPE primer curso de Educación Primaria.

Nota: la línea horizontal representa el valor promedio obtenido por el grupo

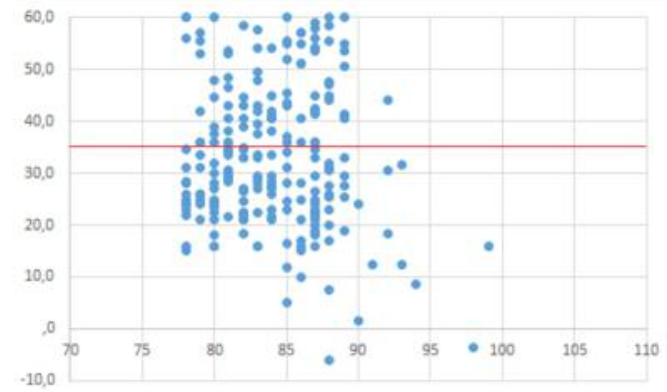

En la Figura 4 se representan los resultados obtenidos por el grupo de estudiantes de primer curso de Educación Primaria. Los datos muestran que las puntuaciones obtenidas oscilan entre -6 y 60 puntos, no existiendo una puntuación techo (únicamente el 2.7\% alcanzan la máxima puntuación). Además, el $55.16 \%$ de los estudiantes han obtenido puntuaciones inferiores a la media.

Si se analizan los resultados correspondientes al grupo de alumnos de segundo curso de Educación Primaria, se observa que el 45.9\% de los participantes obtienen puntuaciones inferiores a la media, poniendo de manifiesto también que los procesos de reconocimeinto de la palabra escrita es una habilidad desarrollada con relativa fluidez en los estudiantes sin ningún tipo de dificultad (Figura 5).

Figura 5. Resultados PIPE segundo curso de Educación Primaria Nota: la línea horizontal representa el valor promedio obtenido por el grupo

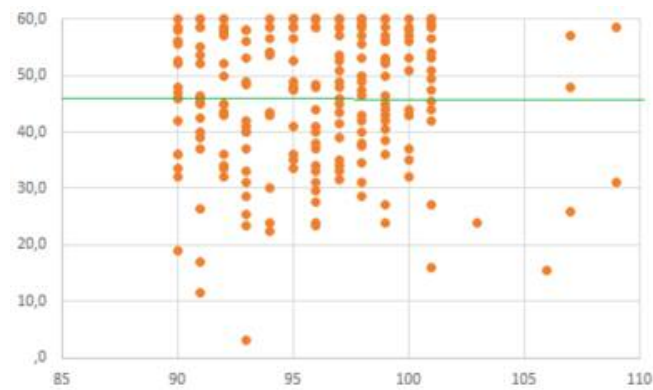


Otra de las cuestiones que ponen de manifiesto los resultados de la prueba PIPE es la evolución en la adquisición de los procesos de reconocimiento de la palabra escrita, es decir, es un proceso cada vez más preciso (se cometen menos errores) y más automático (se reconocen palabras escritas sin consumir demasiados recursos cognitivos básicos de atención y memoria) (Figura 6).

Figura 6. Puntuaciones Medias prueba PIPE por curso

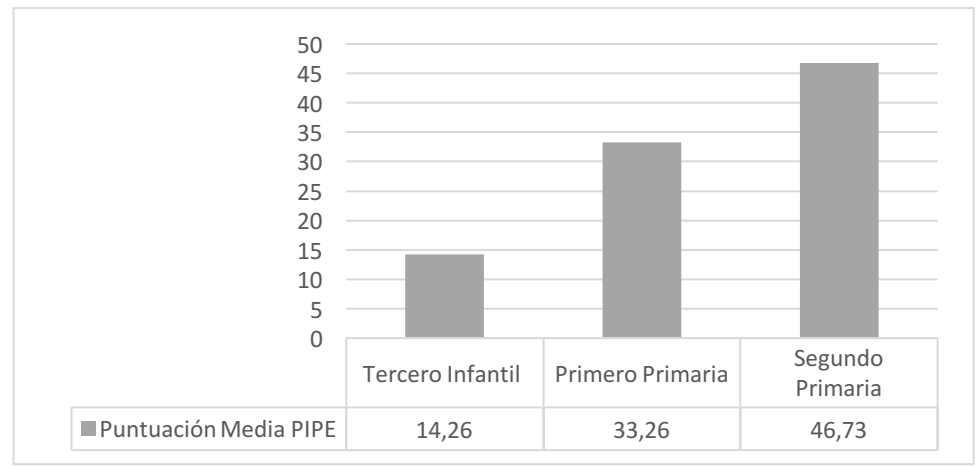

\section{DISCUSIÓN Y CONCLUSIONES}

La prueba presentada en este trabajo contribuye, por un lado, a ampliar la oferta de pruebas existentes en castellano para la evaluación de los procesos específicos de la lectura, y por otro, a evaluar la fluidez lectora en los procesos de reconocimiento de la palabra escrita, pues, en un tiempo máximo de tres minutos, el estudiante debe completar el mayor número de ítems (velocidad lectora) sin cometer o cometiendo el menor número de errores (precisión). Los resultados aquí presentados no permiten extraer conclusiones generalizables a toda la población española de Educación Infantil y Primer y Segundo curso de Educación Primaria puesto que son pocos los estudiantes evaluados, podrán aportarse datos más concluyentes una vez finalizado el proceso de evaluación por parte de la Consejería de Educación de la Junta de Castilla y León. Sin embargo, sí pueden exrtaerse importantes implicaciones educativas en la detección de dificultades de aprendizaje de la lectura desde las primeras etapas escolares: prevenir e intervenir de forma temprana en las dificultades de aprendizaje. Estas implicaciones apoyan las investigaciones realizadas por Rodríguez y Jiménez (2014), quienes apuestan por modelos proactivos de intervención frente a modelos reactivos (aquellos que esperan a que exista un fracaso para intervenir).

\section{AGRADECIMIENTOS}

Este trabajo se enmarca dentro del proyecto de investigación I+D+i "Elaboración de un programa de enseñanza de habilidades morfosintácticas para estudiantes sordos: Efectos sobre las estrategias de lectura de frases" (ref. EDU2014-52739-P), Ilevado a cabo por el Grupo de Investigación COMPLyDIS de la Universidad de Salamanca (https://complydis.usal.es).

\section{REFERENCIAS BIBLIOGRÁFICAS}

Alegría, J. (1985). Por un enfoque psicolingüístico del aprendizaje de la lectura y sus dificultades. Infancia y Aprendizaje, 29 (8), 79-94.

Alegría, J. (2003). Deafness and Reading. En T. Nunes y P. Bryant (Eds.). Handbook of Children s 
Literacy. Dordrescht: Kluwer Academic Publishers

Alegría, J. (2006). Por un enfoque psicolingüístico del aprendizaje de la lectura y sus dificultades, 20 años después. Infancia y Aprendizaje., 29 (1), 93-111. doi: 10.1174/021037006775380957. Alegría, J., Carrillo, M., y Sánchez, E. (2005). La enseñanza de la lectura. Investigación y Ciencia, 340, 6-15.

Carrillo, M.S. y Marín, J., (1997). Prueba de Eficiencia Lectora (PEL). En A. Cuadro, D. Costa, D. Trias, y P. Ponce de León (2009). Evaluación del nivel lector. Manual técnico del test de Eficacia Lectora (TECLE) (pp 20-38). Uruguay: Prensa Médica Latinoamericana.

Castejón, L., González-Pumariega, S., y Cuetos, F. (2011). Adquisición de la fluidez en la lectura de palabras en una muestra de niños españoles: un estudio longitudinal. Infancia y Aprendizaje, 34, 19-30. doi:10.1174/021037011794390139.

Castejón, L., González-Pumariega, S., y Cuetos, F. (2015). El desarrollo de la fluidez en la lectura de palabras en educación primaria: un seguimiento Iongitudinal de seis años. Infancia y Aprendizaje: 38 (4), 842-841. doi:10.1080/02103702.2015.1076266.

Cuetos, R., Ramos, J. L., Ruano, E., y Arribas, D. (2007). Evaluación de los procesos lectores revisada -PROLEC R. Madrid: TEA Ediciones.

Cuetos, F. y Valle, F. (1988). Modelos de lectura y dislexias. Infancia y Aprendizaje, 44, 3-19.

Coltheart, M. (2005). Modeling Reading: The Dual-Route Approach. En Snowling, M. J. y Hulme, C. The Science of Reading: A Handbook. Oxford: Blackwell Publishing. (6-23).

Coltheart, M. (2006). Dual route and connectionist model of reading: an overview. London Review of Education, 4(1), 5-17. doi: 10.1080/13603110600574322.

Coltheart, M., Curtis, B., Atkins, P. y Haller, M. (1993). Models of reading aloud: Dual-route and parallel-distributed-processing approaches. Psychological review, 100(4), 589-608.

Coltheart, M. Rastle, K. Perry, C., Langdon, R. y Ziegler, J. (2001). DCR: A Dual Route Cascaded Model of Visual Word Recognition and Reading Aloud. Psychological Review. 108(1), 204-256.

Defior, S. (2014). Procesos implicados en el reconocimiento de las palabras escritas. Aula, 20, 25-44.

Domínguez, A.B., Alegría, J., Carrillo, M., y Soriano, J. (2013). PEALE. Pruebas de Evaluación Analítica de Lengua Escrita. Universidad de Salamanca. Número de asiento registral: 00/2013/4067.

Domínguez et al. (2014). Elaboración de un programa de enseñanza de habilidades morfosintácticas para estudiantes sordos: efectos sobre las estrategias de lectura de frases. Investigación financiada por el MICINN (proyecto EDU2014-52739-P).

Gough, P. B., y Tunmer, W. E. (1986). Decoding, reading, and reading disability. Remedial and Special Education, 7(1), 6-10.

Hoover, W. A., y Gough, P. B. (1990). The simple view of reading. Reading and writing, 2(2), 127160.

Jiménez, J. E., Luft Baker, D., Rodríguez, C., Crespo, P., Artiles, C., Alfonso, M., ... \& Suárez, N. (2011). Evaluación del progreso de aprendizaje en lectura dentro de un Modelo de Respuesta a la Intervención (RtI) en la Comunidad Autónoma de Canarias. Escritos de Psicología (Internet), 4(2), 56-64. doi: 10.5231/psy.writ.2011.1207.

Junta de Castilla y León 2015- 2020. (2015). II Plan de Atención a la Diversidad Recuperado de www.jcyl.es/junta/cp/Proyecto_Plan_Atencion_Diversidad.pdf.

Martínez, J.A., y García, E. (2004). Diccionario de Frecuencias del Castellano Escrito en niños de 6 a 12 años. Servicio de publicaciones de la Universidad Pontifica de Salamanca.

Morais, J. (1994). L Art de lire. Paris. OdileJacobs. Traducción castellana, El arte de leer (1998) 
Madrid: Visor.

National Institute of Child Health and Human Development. (2000). Report of the National Reading Panel. Teaching children to read: An evidence-based assessment of the scientific research literature on reading and its implications for reading instruction (NIH Publication No. 00-4769). Washington, DC: U.S. Government Printing Office.

Perfetti C. A., y Sandak, R. (2000). Reading optimally builds on spoken language: implications for deaf readers. Journal of Deaf Studies and Deaf Education, 5(1), 32-50. doi: 10.1093/deafed/5.1.32.

Ripoll, J.C. y Aguado, G. (2015). Enseñar a leer. Cómo hacer lectores competentes. EOS: Madrid.

Rodríguez, C., y Jiménez, J. E. (2014). Definición, prevención y diagnóstico de la dificultad de aprendizaje. En J. N. García (Coord.). Prevención en dificultades del

desarrollo y del aprendizaje. Madrid: Pirámide.

Toro, J., y Cervera, M. (1980). Test de análisis de lectoescritura. Madrid: Aprendizaje Visor.

Toro, J., Cervera, M., y Ruiz, C. (2002). EMLE: Escala Magallanes de Lectura y Escritura: TALE-2000: manual de referencia. Grupo Albor-Cohs.

Wolf, M., y Bowers, P. G. (1999). The double-deficit hypothesis for the developmental dyslexias. Journal of educational psychology, 91(3), 415-438. 